EPJ Web of Conferences 81, 01026 (2014)

DOI: $10.1051 /$ epjconf/ 20148101026

(C) Owned by the authors, published by EDP Sciences, 2014

\title{
Meson properties from mesic atoms and mesic nuclei
}

\author{
Satoru Hirenzaki ${ }^{1,2, a}$, Natsumi Ikeno ${ }^{3,4}$, Hideko Nagahiro ${ }^{1,5}$, and Yuko Higashi ${ }^{1}$ \\ ${ }^{1}$ Department of Physics, Nara Women's University, Nara 630-8506, Japan \\ ${ }^{2} J$ JARC Branch, KEK Theory Center, Institute of Particle and Nuclear Studies, High Energy Accelerator \\ Research Organization (KEK), Ibaraki 319-1106, Japan \\ ${ }^{3}$ Yukawa Institute for Theoretical Physics, Kyoto University, Kyoto 606-8502, Japan \\ ${ }^{4}$ Department of Physics, Tohoku University, Sendai 980-8578, Japan \\ ${ }^{5}$ Research Center for Nuclear Physics (RCNP), Osaka University, Ibaraki, Osaka 567-0047, Japan
}

\begin{abstract}
Meson properties are believed to have close connection to the fundamental theory, QCD, and have been studied for a long time both theoretically and experimentally. In this report, we study the recent activities in this field and consider the $\eta(958)$ mesic nuclei and the deeply bound pionic atoms. We summarize the possible formation of the $\eta(958)$ mesic nuclei by the $(p, d)$ reactions and report the new possibilities of the spectroscopic study of the pionic atoms using the $\left(d,{ }^{3} \mathrm{He}\right)$ reactions.
\end{abstract}

\section{Introduction}

Meson properties in the extreme conditions such as high density and/or high temperature are believed to provide important information on the aspects of quantum chromodynamics (QCD) symmetry in these conditions [1]. Meson-nucleus systems are very interesting objects in this context since they are used to investigate the meson properties such as their masses and interactions at finite density. For example, the partial restoration of chiral symmetry was concluded by determining the pion weak decay constant $f_{\pi}$ from the observation of deeply bound pionic atoms using the Tomozawa-Weinberg and Gell-Mann-Oakes-Renner relations [2]. In this article, we report briefly the recent research activities of $\eta(958)\left(\eta^{\prime}\right)$ mesic-nucleus and deeply bound pionic atoms.

\section{$2 \eta^{\prime}$-mesic nuclei}

The $\eta^{\prime}$ meson is known to have exceptionally large mass and close connection to the $\mathrm{U}_{A}(1)$ anomaly. For the investigation of the $\mathrm{U}_{A}(1)$ anomaly, the formations of the $\eta^{\prime}$-mesic nuclei have been studied in Ref. [3].

Recently, there have been important developments in theoretical $[4,5]$ and experimental [6] points of view for the study of the $\eta^{\prime}$ mass at finite density. It has been pointed out theoretically that the anomaly effect can contribute to the $\eta^{\prime}$ mass only with the presence of the spontaneous and/or explicit breaking of chiral symmetry $[4,7]$. As a consequence, even if density dependence of the $\mathrm{U}_{A}(1)$ anomaly effects are negligible, a relatively large mass reduction $(\sim 100 \mathrm{MeV})$ of the $\eta^{\prime}$ meson is

ae-mail: zaki@cc.nara-wu.ac.jp 
expected at nuclear density due to the partial restoration of chiral symmetry. The experiment of the ${ }^{12} \mathrm{C}(p, d)$ reaction was performed at GSI very recently [8] based on the theoretical predictions of the spectra [5] and the observed spectra will be reported in near future.

As a future plan, the semi-exclusive measurement of the ${ }^{12} \mathrm{C}(p, d)$ reaction has been proposed at FAIR [8], where charged particles emitted from the $\eta^{\prime}$ absorption in nucleus are observed in coincidence with the forward deuteron to reduce the background and improve the $\mathrm{S} / \mathrm{N}$ ratio. For the evaluation of the advantages of the semi-exclusive measurement, the conversion part of the theoretical $(p, d)$ spectra shown in Fig. 1 and the simulation program JAM [9] have been used. The quantitative results for the improvement of the $\mathrm{S} / \mathrm{N}$ ratio will be reported [10].

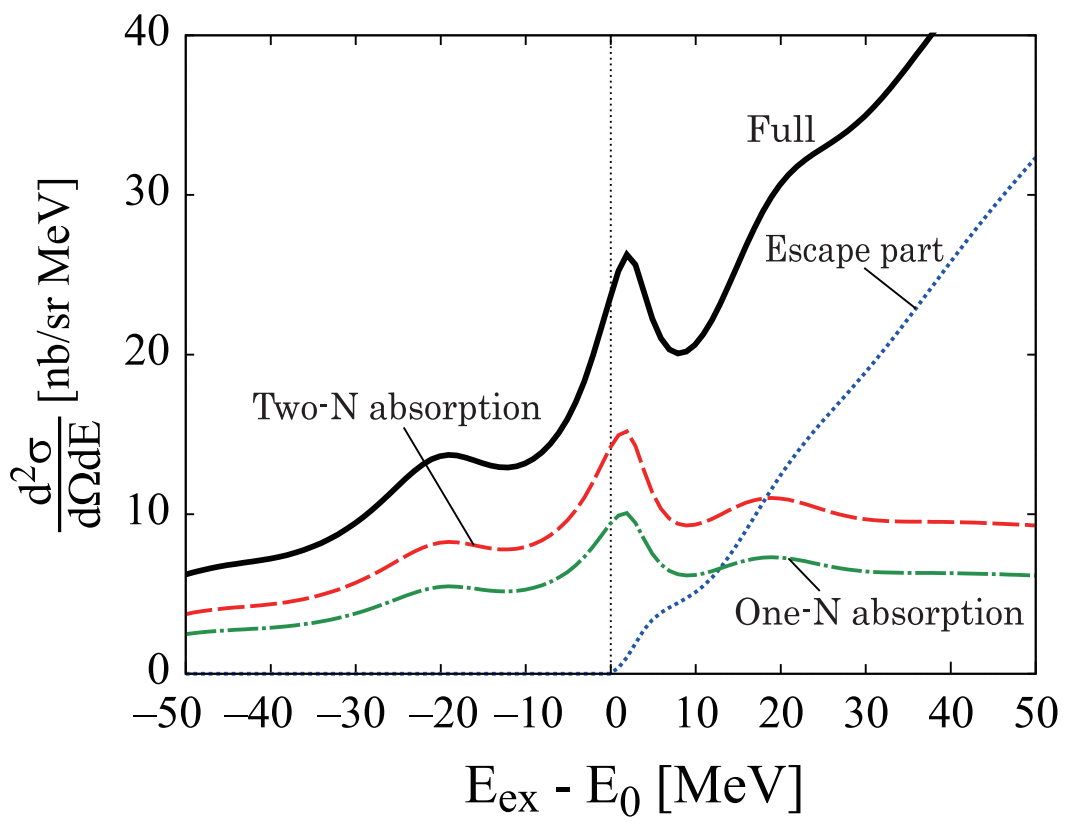

Figure 1. Calculated spectra of the ${ }^{12} \mathrm{C}(p, d){ }^{11} \mathrm{C} \otimes \eta^{\prime}$ reaction for the formation of $\eta^{\prime}$-nucleus systems with proton kinetic energy $T_{p}=2.5 \mathrm{GeV}$ and deuteron angle $\theta_{d}=0 \mathrm{deg}$. as functions of the excited energy $E_{e x}$ reported in Ref. [5]. $E_{0}$ is the $\eta^{\prime}$ production threshold. The $\eta^{\prime}$-nucleus optical potential is evaluated in Ref. [11], which corresponds to the $\eta^{\prime}$ scattering lengths $\mid a_{\eta^{\prime}} p=1.0 \mathrm{fm}$. The thick solid line shows the total spectrum. The dotted, dashed and dot-dashed lines show the escape and conversion parts of the spectrum as indicated in the figure.

\section{Deeply bound pionic atoms}

Within various meson-nucleus systems, the pionic atom is the best system to perform accurate spectroscopic studies, since the width of the pionic atom is smaller than other meson-nucleus systems. In addition, we have well established theoretical and experimental techniques. So far, based on the theoretical predictions [12] there exist four sets of successful experimental results for the formation 
of deeply bound pionic atoms in the $\left(d,{ }^{3} \mathrm{He}\right)$ reaction [2, 13-18]. After these experiments, Ikeno et $a l$. have developed further theoretical studies of the formation spectra of the pionic atoms in order to find out the appropriate procedure to obtain more precise information on chiral symmetry restoration from the observables [19-22]. New experiment at RIBF/RIKEN was also performed and the observed spectra will be reported in near future [23].

In Fig. 2, we show the latest theoretical predictions of the $\left(d,{ }^{3} \mathrm{He}\right)$ reaction on ${ }^{117} \mathrm{Sn}$ target [21]. The target nucleus ${ }^{117} \mathrm{Sn}$ has the odd-number neutron, and hence, the pionic atoms are populated in the even-even nucleus ${ }^{116} \mathrm{Sn}$ in the final state where we do not need to worry about the residual interaction effects reported in Refs. [24, 25], which could cause additional difficulties to deduce information on chiral condensate from data. The experiment of the ${ }^{117} \mathrm{Sn}\left(d,{ }^{3} \mathrm{He}\right)$ reaction was also performed at RIBF/RIKEN [23].

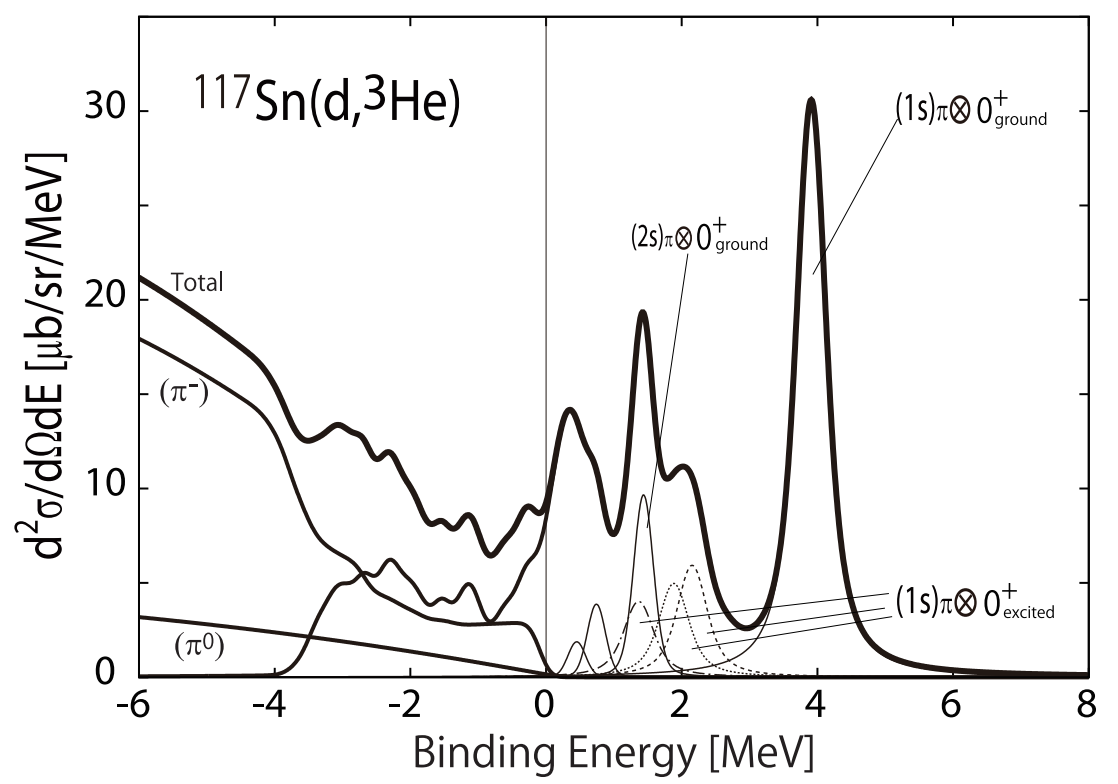

Figure 2. Calculated spectrum for the formation of pionic states in the ${ }^{117} \mathrm{Sn}\left(d,{ }^{3} \mathrm{He}\right)$ reaction at $\theta_{d H e}^{l a b}=0$ deg. plotted as function of the pion binding energy reported in Ref. [21]. The incident deuteron kinetic energy is fixed at $T_{d}=500 \mathrm{MeV}$. The total spectrum is shown by the thick solid line. We show separately the contributions of the bound pionic state formation and the quasi-free $\pi^{-}$and $\pi^{0}$ production by thin solid lines. The dominant subcomponents are also shown in the figure with quantum numbers indicated as $\left[(n \ell)_{\pi} \otimes J^{P}\right]$. The instrumental energy resolution is assumed to be $300 \mathrm{keV} \mathrm{FWHM.} \mathrm{The} \mathrm{contribution} \mathrm{of} \mathrm{the} \pi^{0}$ quasi-free production is assumed to be the same as that of the ${ }^{122} \mathrm{Sn}\left(d,{ }^{3} \mathrm{He}\right)$ reaction, except for the shift of the threshold energy [21].

\section{Summary}

We have reported briefly the recent research activities of $\eta^{\prime}$ mesic-nucleus and deeply bound pionic atoms. Especially, we have studied the latest theoretical results on the conversion ( $\eta^{\prime}$ absorption) contribution of the $\eta^{\prime}$ formation reaction [5] and the ${ }^{117} \mathrm{Sn}\left(d,{ }^{3} \mathrm{He}\right)$ reaction for the pionic atom formation [21] with some explanation of their importance. We believe that these results provide a good 
motivation for further experiments and help significantly developments of the studies of the symmetry aspects of the strong interaction at finite density.

\section{References}

[1] T. Hatsuda and T. Kunihiro, Phys. Rep. 247, 221 (1994), and references therein.

[2] K. Suzuki et al., Phys. Rev. Lett. 92, 072302 (2004).

[3] H. Nagahiro and S. Hirenzaki, Phys. Rev. Lett. 94, 232503 (2005); H. Nagahiro, M. Takizawa, and S. Hirenzaki, Phys. Rev. C74, 045203 (2006).

[4] D. Jido, H. Nagahiro, and S. Hirenzaki, Phys. Rev. C85, 032201(R) (2012).

[5] H. Nagahiro et al., Phys. Rev. C87, 045201 (2013).

[6] K. Itahashi et al., Prog. Theor. Phys. 128, 601 (2012).

[7] S. H. Lee and T. Hatsuda, Phys. Rev. D54, R1871 (1996).

[8] K. Itahashi, H. Fujioka, Y. Tanaka, private communication.

[9] Y. Nara et al., Phys. Rev. C61, 024901 (2000).

[10] Y. Higashi et al., in preparation.

[11] H. Nagahiro, S. Hirenzaki, E. Oset, and A. Ramos, Phys. Lett. B709, 87 (2012).

[12] T. Yamazaki, S. Hirenzaki, R. S. Hayano, and H. Toki, Phys. Rep. 514, 1 (2012).

[13] T. Yamazaki et al., Z. Phys. A355, 219 (1996).

[14] H. Gilg et al., Phys. Rev. C62, 025201 (2000).

[15] K. Itahashi et al., Phys. Rev. C62, 025202 (2000).

[16] H. Geissel et al., Phys. Rev. Lett. 88, 122301 (2002).

[17] K. Itahashi et al., EPJ Web Conf. 37, 01013 (2012).

[18] S. Itoh, Doctor Thesis, University of Tokyo (2011).

[19] N. Ikeno et al., Prog. Theor. Phys. 126, 483 (2011).

[20] N. Ikeno, H. Nagahiro, and S. Hirenzaki, Eur. Phys. J. A47, 161 (2011).

[21] N. Ikeno et al., Prog. Theor. Exp. Phys. 2013, 063D01 (2013).

[22] N. Ikeno et al., Submitted to Prog. Theor. Exp. Phys. (2014).

[23] K. Itahashi, T. Nishi, private communication.

[24] S. Hirenzaki et al., Phys. Rev. C60, 058202 (1999).

[25] N. Nose-Togawa et al., Phys. Rev. C71, 061601(R) (2005). 